\title{
EDITORIAL
}

\section{Editorial—Rheumatology in Africa}

\author{
Ade Adebajo $^{1}$ (D) Asgar Kalla ${ }^{2} \cdot$ Mohammed Tikly $^{3}$
}

Received: 15 July 2021 / Revised: 15 July 2021 / Accepted: 15 July 2021 / Published online: 28 July 2021

(c) International League of Associations for Rheumatology (ILAR) 2021

This special collection on rheumatology in Africa reflects a new dawn for rheumatology on the continent as well as the continuing strong support of Clinical Rheumatology for the development of African rheumatology [1]. In contrast to earlier reports, rheumatological conditions are now not considered to be rare in Africa and the articles within this collection reflect this. With increasing globalisation, even the rheumatological care needs of migrants from SubSaharan Africa, living in the West, are receiving attention as discussed in this special collection. There is also a wider than hitherto appreciated range of these disorders on the African continent. Thus, this special collection includes articles on psoriatic arthritis, gout and osteoporosis in Africa. In general, these conditions have been less often reported from Africa, than rheumatoid arthritis and systemic lupus erythematosus.

This increasingly recognised range of rheumatological disorders on the African continent requires that rheumatological care, education and research in Africa need ramping up, as one of the articles in this collection addresses. Fortunately, these issues are being tackled vigorously, particularly by the African League Against Rheumatism (AFLAR), in collaboration with international partners including ILAR, EULAR, ACR and BSR. The number of trained rheumatologists and other rheumatology health care professionals is increasing rapidly, accompanied by efforts to establish currently available resources, in order to address the resource gaps.

Not only is rheumatology experiencing a new dawn on the African continent, the contribution of African rheumatologists internationally is being recognised and rewarded by organisations such as the American College of Rheumatology (for example, Professors Femi Adelowo and Girish Mody) and the British Society for Rheumatology. Even the current coronavirus pandemic has not slowed the development of African rheumatology. Rather, the pandemic has accelerated these developments [2].

These are exciting times for African rheumatology with regard to service provision, research and education. Ultimately, this will yield many benefits for patients with rheumatic and musculoskeletal disorders in Africa.

\section{Declarations}

Disclosures None.

\section{References}

1. Genga EK, Oyoo O, Espinoza LR, Adebajo A (2017) African Journal of Rheumatology; enhancing the visibility of rheumatology in Africa. Clin Rheumatol 36:2167-2168

2. Akintayo R, Kalla A, Adebajo A (2020) COVID-19 and African rheumatology: progress in adversity. Lancet Rheumatol 20:30347-30357

Publisher's note Springer Nature remains neutral with regard to jurisdictional claims in published maps and institutional affiliations.

Ade Adebajo

a.o.adebajo@sheffield.ac.uk

1 Faculty of Medicine, Dentistry and Health, University of Sheffield, Sheffield, UK

2 Department of Medicine, University of Cape Town, Cape Town, South Africa

3 Department of Medicine, Chris Hani Baragwanath Academic Hospital, Soweto, South Africa 